\title{
Factors influencing truth-telling by healthcare providers to terminally ill cancer patients at Ocean Road Cancer Institute in Dar-es-Salaam, Tanzania
}

\author{
R Athanas, ${ }^{1}$ Dip Philo, BA Theo, MBE; F Gasto, ${ }^{2}$ PhD; S J Renatha, ${ }^{1,3}$ MD, MMed, MBE \\ ${ }^{1}$ School of Public Health and Social Sciences, Department of Bioethics and Health Professionalism, Muhimbili University of Health and Allied Sciences, \\ Dar es Salaam, Tanzania \\ ${ }^{2}$ School of Public Health and Social Sciences, Department of Development Studies, Muhimbili University of Health and Allied Sciences, Dar es Salaam, \\ Tanzania \\ ${ }^{3}$ Bioethics Department, Muhimbili National Hospital, Dar es Salaam, Tanzania
}

Corresponding author: R Athanas (mukhotya@gmail.com)

Background. Widely accepted international clinical ethics standards and Tanzania's national cancer treatment guidelines mandate that healthcare providers give patients the information they want or need in an understandable way. However, adherence to these standards is low, particularly in developing countries. In Tanzania, little is known regarding the views and practice of disclosure of diagnosis and prognosis to cancer patients.

Objective. To examine the factors influencing truth-telling by healthcare providers to terminally ill cancer patients at Ocean Road Cancer Institute (ORCl) in Dar-es-Salaam, Tanzania.

Methods. We conducted semi-structured interviews with a purposive sample of healthcare providers $(n=13)$ and terminally ill cancer patients $(n=8)$ in English and Swahili. The interviews were recorded, transcribed verbatim and translated into English.

Results. The study findings show an unsatisfactory experience of truth-telling according to international clinical standards. Many healthcare providers emphasised barriers including: limited communication skills; limited time; a high volume of patients; and lack of privacy for communication. Patients' preferences and readiness to receive and accept information, as well as family collusion, were identified as barriers, whereas communalism was identified as a facilitator to truth-telling.

Conclusion. We identified several barriers and facilitators of truth-telling that may be targeted in order to promote high-quality communication at $\mathrm{ORCl}$. Our results support advocacy for improvements such as: training for clinical communication skills and information sharing; space designated to enhance privacy; deliberate efforts to employ enough providers to care for the increasing volume of patients; and need for counselling session with patients and family members prior to clinical information disclosure.

S Afr J Bioethics Law 2020;13(2):108-113. https://doi.org/10.7196/SAJBL.2020.v13i2.706

The accepted international medical ethical code recommends that patients should not be denied information by healthcare providers pertaining to their own health and life. ${ }^{[1]}$ Likewise, Tanzania's national cancer treatment guidelines mandate that healthcare providers give patients the information they want or need in an understandable way. ${ }^{[2]}$ This is because a patient has a fundamental right to medical information, ${ }^{[3]}$ and informed consent affirms this. ${ }^{[4,5]}$ Disclosure of clinical information has to do with total openness and transparency in revealing clinical situations, treatments and the possible outcomes to a patient. It includes the expected duration, function and description of the course of the disease, such as progressive decline, intermittent crisis and unpredictable crisis. ${ }^{[6]}$ However, whether to tell the truth or not causes a dilemma for some medical practitioners. ${ }^{[7]}$ Withholding the truth is a form of medical paternalism, ${ }_{1}^{[8]}$ while medical ethics pertaining to truth-telling supports respect for the autonomy of a patient, and thus rejects paternalism. ${ }^{[9]}$ The truth-telling dilemma in cancer treatment contexts is complex, in the sense that merely telling a patient 'You have cancer' creates an ethical problem, as this may lead to loss of hope. ${ }^{[10]}$ Truth-telling is important in the sense that failure to tell the truth to terminally ill cancer patients results in a lack of advance directives, inadequate psychological and spiritual preparation and family misunderstandings. In addition, failure to tell the truth to patients can cause all sorts of anxiety, tensions and despair to patients, such that they may die while unprepared as a result of information denial.

The practice regarding disclosure of cancer diagnosis and prognosis varies globally; in the USA, $83 \%$ of a sample of 219 healthcare providers reported that they would opt to tell the truth. ${ }^{[11]}$ The great majority of healthcare providers (79\%) in Kuwait would withhold the truth if the patient's family requested them to do so. ${ }^{[12]}$ In Saudi Arabia, $75 \%$ of healthcare providers tell close relatives rather than the patient. ${ }^{[13]}$ Japanese patients generally surrender moral consent and autonomy to that of their family members. ${ }^{[14]}$

In Ethiopian culture, the honesty and integrity of a healthcare provider are not questioned, whether one decides to tell the truth or not. ${ }^{[15]}$ In Tanzania, little is known about clinical information disclosure to cancer patients, owing to limited evidence available regarding factors influencing the telling or not telling of the truth. However, 
the culture of Tanzania does not allow such information to be given directly to patients, to protect cancer patients from losing hope and experiencing depression. ${ }^{[16]}$ The aim of this study was to explore the factors influencing truth-telling by healthcare providers to terminally ill cancer patients at the Ocean Road Cancer Institute in Dar-es-Salaam, Tanzania. Specifically, the study aimed to examine: (i) healthcare provider-related factors; (ii) patient-related factors; and (iii) sociocultural factors influencing truth-telling in cancer treatment settings.

\section{Methods}

This study was conducted at the Ocean Road Cancer Institute (ORCI) in Dar-es-Salaam, Tanzania. The hospital was established in 1996 as a national referral centre for cancer treatment, and provides free care to 5400 new patients per year, including services such as radiotherapy and chemotherapy. The authors purposively selected the area because it is the only specialised facility for cancer treatment in Tanzania. Given its nature, the ORCl serves many cancer patients and hosts a number of specialised cancer healthcare providers. It is located in Dar es Salaam, the largest city in Tanzania. The ORCI provides cancer care services for all types of cancer to both adult outpatients and inpatients. Approximately 257 patients are treated on a daily basis. It has a total number of 19 specialists, of whom 11 are females and 8 are males.

Data were extracted from two purposively sampled groups of key informants. ${ }^{[17]}$ Healthcare providers included oncologists, palliative care specialists and nurses $(n=13)$. All healthcare providers who participated in this study were purposively selected because they were knowledgeable, highly experienced with at least 2 years of work experience in the oncology field and are directly involved in treating terminally ill cancer patients. Their names and contact details were obtained from the head of the research and training unit. Some of them were contacted physically, and others were telephoned to ask for their participation. Those who agreed were free to decide on the time, date and place of interview.

We selected patients who were at a terminal stage, with end-oflife $\leq 6$ months, admitted to a special room receiving only palliative treatment, but who could still communicate $(n=8)$. The reason for including patients in this study was to find out exactly what they need to know about their illness, and to what extent (how much), and when. During patients' selection, a list of names was obtained from the palliative care specialists and nurses in charge, with permission from the heads of the wards. On each day of the interview, patients were approached by the researcher or an assistant, and were asked to participate voluntarily in the study. Each group was assigned its own interview guides. The interview guides were pilot tested at Muhimbili National Hospital (MNH) with healthcare providers and patients, and questions were rephrased in line with the challenges encountered during the piloting phase. Each interview guide contained openended questions, with subsequent probing questions. The questions were tailored to answer the three study objectives regarding factors that influence truth-telling by healthcare providers to terminally ill cancer patients at the ORCl. Interviews were conducted by the researchers with help from two research assistants adequately qualified in caring for terminally ill cancer patients. The interviews were conducted in a separate isolated room so as to maintain the privacy of key informants and confidentiality of the information they shared. Moreover, the researcher ensured that questions were fully exhausted by participants, and data collection stopped when all the themes became saturated. With permission from key informants, interviews were audiorecorded. Furthermore, field notes were taken for each interview to smoothly facilitate data analysis.

\section{Data analysis}

Data transcription and translation were done by the researchers with help from two research assistants. Thematic analysis using an inductive approach was used to analyse the collected information. The use of an inductive approach aims to ensure that the emerging themes are strongly linked to the data themselves, and that they are not imposed by the researcher. The first author coded the data, and all authors participated in analysing the data manually through reading and re-reading the transcripts to ensure a clear and general understanding of the emerging concepts. Themes were then sought out from the general lists of the created codes (Table 1). The whole process of analysis was iterative. Further scrutiny was carried out by returning to the interview transcripts to identify their similarities and differences, to ensure that the identified themes formed coherent patterns. Finally, we clustered the subthemes and the themes, and presented them with supporting quotations that describe the meaning underpinning each theme.

\section{Ethical considerations}

We sought and were granted ethical clearance from Muhimbili University of Health and Allied Sciences (MUHAS) Ethical Review Committee to conduct the study (ref. no. DA.287/298/01A/07). Permission to conduct the research was also requested from the ORCl, where the study participants work. Written consent was obtained

Table 1. Condensed test, codes, subthemes and themes

\begin{tabular}{lll}
\hline Condensed meaning units ('test') & Codes & Subthemes \\
\hline $\begin{array}{l}\text { 'Here there are gaps due to aspects like communication } \\
\text { skills' }\end{array}$ & Skill level & Lack of communication skills \\
'Usually there is no time to do that' & Management barrier & Limited time \\
'You need to have a conducive setting for cancer & Structural limitation & Lack of privacy \\
information sharing' & & Provider-level factor \\
'Here patients are so many' & Hospital limitation & High volume of patients \\
$\begin{array}{l}\text { 'I need to hear good news that I am curing and going } \\
\text { home tomorrow' }\end{array}$ & Choice & Patient preferences \\
'I feel not ready for information' & Willingness & Provider-level factor \\
'Our culture does not allow us' & Cultural limitation & Patient readiness \\
Pressure from family members & Relatives involved & Communalism \\
& & Family collusion
\end{tabular}


from participants after explaining to them the study objectives, methodology and benefits. Participants were also assured of the confidentiality of all the information they disclosed to the researchers.

\section{Results}

Upon analysis of the study findings, seven subthemes were generated, and grouped into three main themes, namely healthcare provider-related, patient-related and sociocultural factors influencing truth-telling. The subthemes generated under healthcare providers were: limited communication skills; limited time; lack of privacy for communication; and high volume of patients. The subthemes under patients were: patients' preferences; and readiness to receive and accept information. Communalism and family collusion were the subthemes under the sociocultural theme.

\section{Limited communication skills}

Truth-telling to patients was generally perceived by most healthcare providers as the key role in the oncology field. The main issue was how to start communicating the bad news to patients. The task tends to place healthcare providers in a dilemma about whether to communicate the crucial information or not. It was further reported that truth-telling should never be reduced to merely telling patients that they have cancer. Participants said that it should go beyond this, as a quotation from one interviewee illustrates:

Here we have I think a big gap in truth-telling due to so many aspects like communication skills. We may be able to provide symptoms managements to a certain good number of patients, but what is happening afterward is a big question mark that should be addressed.' MD 010, oncologist

\section{Limited time}

Limited time was one of the system-level barriers to information disclosure. The healthcare providers interviewed for this study reported that they have minimal time available to afford a thorough discussion with a patient. Time is always not a doctors' friend, as claimed by one of the participants, who cited it as one of the reasons why healthcare providers sometimes receive patients who are not aware of their diagnosis:

'A doctor may not have enough time to tell the truth to a patient due to limited time. You find yourself with so many patients to attend to in a day such that you give just a fraction of a minute to each patient of which it is not enough to digest all of the information.'MD 008, oncologist

\section{Lack of privacy for communication}

It was reported by healthcare providers that providing complete information to patients requires a conducive setting where each party has the freedom of sharing and listening. The existing settings of the hospital do not provide rooms that guarantee adequate privacy to facilitate effective disclosure of medical information. The participants reported being ethically failed by a lack of hospital infrastructure such as private offices, rooms for private counselling and a suitable place for information sharing:

'First of all, the issue of breaking cancer information is crucial. You need to have a physical support like a private office, room and a good place to talk whereby a patient would feel comfortable and able to hear and cope with the news.' MD 006, oncologist

\section{High volume of patients}

A high volume of cancer patients who come to seek health services on a daily basis was cited as a barrier to truth-telling at the hospital. It was reported by participants that the ever-increasing number of cancer patients relative to the availability of healthcare providers tends to limit adequate time available for the comprehensive disclosure of medical information to patients:

'Here patients are so many to the extent in the sense that those in charge of giving the information do not have enough time to sit with a patient and share the information wholly.' MD 009, oncologist

\section{Patients' preferences about information}

Key findings further showed that patient's preferences about access to information also influence the truth-telling process. This was cited as a barrier to truth-telling because imposing information on an unprepared and unwilling patient is not an act of respect for autonomy. Both healthcare providers and patients interviewed for this study reported the preferences of patients regarding medical information as an important factor that featured in the truth-telling process in oncology, as one participant made clear:

'From my experience, patients also influence the truth-telling process in oncology. This is because some of them do not want to hear certain kinds of information, especially that which bears a negative element in it. Some prefer to hear information that brings healing hope.' MD 011, oncologist

This was also confirmed by some patients who were interviewed about the type of information that they would want to receive from healthcare providers. One of the participants reported that he would like to receive good news on how his condition was improving:

II need to receive good news, that I am recovering, that my condition is improving, that I am getting better and going home tomorrow. This is the kind of information I would like to hear from healthcare providers. I do not like to hear the news that break my heart, news which makes me become afraid of and put me into fear.'PT 06, patient

\section{Patients' readiness to receive information}

Truth-telling was said to be influenced by the psychological status and ability of the patient to handle the information. Some of the study participants interviewed for this study said that patients are sometimes not ready to receive medical information. One of the healthcare providers said :

'Truth-telling depends on the readiness of a patient. You have to look at the patient's facial expression and conclude that sometimes patients cannot handle information comprehensively. In this regard as an oncologist, you are obliged to break the news slowly, little by little, while monitoring the psychological readiness of your patient.' MD 008, oncologist

Another patient interviewed for this study acknowledged the influence of his psychological state in receiving medical information, saying:

'Sometimes I feel not ready for my medical information, because I believe there is bad news and I do not want to hear such news that break my heart into pieces. I am ready to hear the information that 
give me hope, encourage my faith, that the medications will cure me.' P02, patient

\section{Communalism}

The findings of this study revealed the influence of cultural communication style as a barrier to truth-telling by healthcare providers to terminally ill cancer patients. It was reported that when the issue of discussing medical information arises, in most cases, many African cultures, including Tanzanian, emphasise communalism rather than the interests of the individual patient. This encourages withholding information from the patient. One participant said:

'We usually discuss the information with family members in my culture, so as to protect the patients, because if the patient knows it is likely that he or she will lose hope, experience depression, fear, and eventually die.' MD 010, oncologist

\section{Family collusion}

The majority of healthcare providers who were interviewed for this study reported the influence of family collusion in providing medical information to their cancer patients. The key findings were further that family members often prevent healthcare providers from sharing information with patients. Most patients interviewed also cited the role of their families in receiving medical information. One of the healthcare provider participants said:

'Family members can interfere and prevent you from communicating the news directly to the patients. Many times, I get pressure from family members who would approach me asking not to disclose the information to their patients. We have to respect family members in disclosing the information.' MD 010, oncologist

\section{Discussion}

Healthcare provider participants revealed several factors that influence their disclosure of medical information to terminally ill cancer patients. This discussion is based on the objectives of the study, which aimed to examine the healthcare provider-related, patient-related and sociocultural factors influencing truth-telling to terminally ill cancer patients. The discussion that follows is based on the findings obtained from the 21 participants of this study (13 healthcare providers and 8 terminally ill cancer patients).

\section{Provider-level factors}

Many participants emphasised the barriers discussed in this section.

\section{Limited communication skills}

The findings revealed limited communication skills among healthcare providers in discussing medical information with cancer patients. The findings show how information about cancer diagnosis and treatment overwhelms healthcare providers in terms of their own feelings and emotions as they communicate bad news to patients. The fear of a patient's reaction to the news is one factor that limits and weighs down a provider's decision as to whether to discuss clinical information. It puts healthcare providers into a dilemma regarding whether to communicate the information or not, because, as has been discussed, telling a patient that his or her cancer is at a late stage is a difficult task, since it tends to cause feelings of terror. ${ }^{[18]}$ This is due to health practitioners' limited communication skills and information-sharing methods, as well as the complex nature of the disease itself. ${ }^{[19]}$ This implies that as a result of reflecting on patients' reaction to cancer information, healthcare providers find that they have little that they can discuss with patients, consequently making truth-telling an even harder task for providers to undertake. Healthcare providers' limited communication skills in discussing clinical information with their patients in the oncology field has been found to compromise the entire truth-telling and general information-sharing process with their patients. ${ }^{[20]}$

\section{Limited time}

This study showed that having insufficient time hinders truthtelling to patients. The interviewed healthcare providers reported themselves as having minimal time available to afford a thorough discussion with a patient. ${ }^{[2]]}$ Each patient is given a very short amount of time, which is never enough to digest all the information one may receive. This implies that truth-telling to patients requires sufficient time to discuss and share medical information. The impact of time in healthcare has also been documented elsewhere, and is said to greatly affect communication. ${ }^{[22]}$

\section{Lack of privacy for communication}

Our study shows that a lack of adequate facilities, such as offices or private rooms, hinders information sharing among healthcare providers and patients. It was reported by most of the interviewed healthcare providers that disclosing complete information to patients requires a conducive setting, where each party involved in the truth-telling process has freedom to share and to listen carefully. This is not the case in the existing settings, which do not provide enough room for privacy to allow for the effective disclosure of medical information. Participants admitted being ethically failed by the lack of infrastructure conducive to information sharing. This implies that deciding to disclose information in inappropriate settings violates privacy, which discourages practitioners from sharing information honestly with patients. A previous study has shown that protection of patient information is part of core professional ethics, and it is clearly understood that such protection is only possible if there are adequate facilities to ensure privacy at health facilities. ${ }^{[23]}$

\section{High volume of patients}

This study showed that the high volume of cancer patients attended at the $\mathrm{ORCl}$ compromises the truth-telling process. It was reported by most healthcare providers that they are obliged to attend to as many patients as possible on a daily basis, because of the everincreasing number of patients reporting at the hospital. The low ratio of healthcare providers to patients tends to limit adequate disclosure of clinical information, due to the time pressure exerted on the providers. The lower the number of patients, the more time would be available for information disclosure. Healthcare providers furthermore reported that it would be unethical to spend more time on one patient than others while there are so many more, some having travelled a long distance, waiting for the service. This implies that the increase in the number of patients on a waiting list results in a decrease in the amount of time available to providers who need it to impart information fully, hence the information is only partially shared. Another study documented the fact that a high increase in the number of new patient cases per month contributed to the majority of healthcare providers becoming reluctant with regard to 
truth-telling, owing to their heavy workload. ${ }^{[24]}$ In addition, healthcare providers know the importance of truth-telling to patients: that it is a basic moral rule in the healthcare profession. Therefore, they understand that not to tell the truth may jeopardise existing staffpatient trust, and lead to a failure of health professionals to respect cancer patients as autonomous individuals. This undermines the patient's capacity for autonomy, and deprives terminally ill patients of their right to a 'good death'.[25]

\section{Patient-level factors}

Patients' preferences and readiness to receive information were identified as both barriers and facilitators to truth-telling, as discussed in the following section.

\section{Patients' preferences about information}

It was found that there are some patients who are willing to hear information that brings them hope of healing, while others would prefer not to hear any information about their illness. These preferences affect the truth-telling process. Healthcare providers reported that they respect these preferences owing to the widely recognised principle of fundamental medical autonomy regarding information, which allows patients the choice to the type of information they would prefer to access. It is worth noting that imposing information on an unprepared and unwilling patient is not an act of respect for autonomy. ${ }^{[26]}$ This implies that in the effort to impart medical information, healthcare providers have a role to play in educating patients, especially those who tend toward resistance, and refuse to receive details about their ailments and treatment options. A study ${ }^{[22]}$ documented patients' preferences to information as a barrier to truth-telling in the oncology field, as it contributes to the variability of attitudes in cancer patients.

\section{Patients' readiness to receive information}

This study revealed that the readiness of a patient to receive medical information influences truth-telling. Most participants interviewed for the research said that truth-telling depends on the psychological readiness of a cancer patient. Healthcare providers are not willing to share too much medical information with patients if they feel that the patient would not be able to handle it. Opting to share information with patients who are unstable and disturbed psychologically may lead such patients to lose hope. This implies that a healthcare provider's decision whether to tell the truth or not is sometimes determined by the psychological condition of the patient they are attending to. This observation was also supported by one of the interviewed patients, who acknowledged his unwillingness to receive any bad news. The patient admitted to only waiting for information that gives hope, encouragement and the promise of absolute cure for his ailment. A study has also made the finding that under grave circumstances, healthcare providers may be forced to deceive patients so as to instill hope in them, adhering to the ethical principle of beneficence. ${ }^{[27]}$ However, it is also recommended in another study that deception generally should not be utilised in everyday medical practice because it disrespects the autonomy of the patient. ${ }^{[28]}$ In medical practice, providers are morally obliged to follow the doctrines of autonomy and beneficence. However, some providers may mistakenly use benevolent deception because it honours the principle of beneficence.
They may lie to the patient allegedly for his or her benefit, especially when believing that telling the actual truth would cause more harm than benefit. ${ }^{[7]}$ It has been mentioned above that while the majority of providers in both developed and developing countries tell the truth about a patient's health condition, the assumption that truth-telling is always beneficial to patients is sometimes criticised. ${ }^{[29]}$ Truth-telling has some side-effects to patient's health, including psychological effects, as some may be overwhelmed by the information that they are in the final moments of their lives, ${ }^{[30]}$ meaning that the decision to accept the truth can hinge on the degree of personal responsibility that health professionals delegate to patients. It is argued that truthtelling is equal to the avoidance of responsibility by providers, who are expected to involve and guide cancer patients in making decisions about their health. However, some physicians present the available options to cancer patients, and expect them to choose, because this avoids blame from patients and their relatives if something goes wrong. ${ }^{[29]}$

\section{Sociocultural factors}

Communalism and family collusion were identified as a facilitator and barrier to truth-telling, respectively, as discussed below.

\section{Communalism}

Cultural communication style was identified as an influence, as Tanzanian culture embraces communal concern rather than individual interests, something that may encourage the withholding of information from a patient by a provider. In this study, it was found that the culture does not allow much information to be given directly to patients, out of the desire to protect patients from depression and loss of hope, which could lead to premature death. The practice can influence coping outcomes, including the ability to absorb the shocks of cancer diagnosis and prognosis. This implies that society, through communication style, is capable of influencing therapeutic outcomes, and this can result in large differences in medical care services, as well as the ability of the society to absorb shocks in terms of cancer information. ${ }^{[3]}$ The influence of cultural communication style that can manipulate the perceptions and attitudes of people on cancer treatments and information sharing, including truthtelling, has also been acknowledged in another study. ${ }^{[32]}$

\section{Family collusion}

Our study shows that family members normally request that providers do not share information with their patients. It was revealed that patients allow this interference as well as the involvement of family members in the truth-telling and decision-making process on their behalf. This shows that family members may play a major role in imparting information and in making medical decisions for patients. It also entails that family members can influence the medical relationship, including truth-telling, between a provider and a patient. ${ }^{[18]}$ Other studies have also reported the influence of family members in the field of oncology on information sharing, including truth-telling. It has been reported that healthcare providers often find themselves in a quandary as to whom, when and how to tell. ${ }^{[8,33]}$ Such a dilemma is complex in the sense that opting to tell the family members amounts to violating the ethical principle of autonomy of a patient, while lying is wrong and immoral and disrespecting the person's autonomy is not ethical. ${ }^{[34]}$ Despite familyrelated barriers to truth-telling, it is worth noting that one of the key 
values to being truthful is associated with respect for the patient as a person who is able to make the correct decisions that benefit their overall health. Furthermore, if patients do not understand the truth, it may lead to a failure of healthcare professionals to respect cancer patients as autonomous individuals. ${ }^{[34]}$

\section{Study limitations and strengths}

The lack of inter-hospital comparison, and the selection of only hospital-based respondents without including a household-based selection of respondents, may contribute to missing the inclusion of ideas from dissatisfied patients who have stopped seeking medical care in public hospitals such as the ORCl. The possibility of social desirability bias in in-depth interviews with healthcare providers may also limit this study. However, the adequate sample size and use of respondents from multiple categories (oncologists, nurses and patients) are the major strengths of this qualitative study. We believe that this study provides valuable insights into the nature of truthtelling to terminally ill cancer patients by healthcare providers.

\section{Conclusions}

The truth-telling process in the oncology field goes beyond merely providing information related to the patient's diagnosis and prognosis. The data in this study support advocacy for improvements in aspects such as training in clinical communication skills and information sharing, space designated to enhance privacy, deliberate efforts to employ enough healthcare providers to care for the increasing volume of patients, and the need to hold counselling sessions with patients and family members prior to clinical information disclosure. Since this study was conducted in just one hospital, further studies could be conducted involving other hospitals and healthcare institutions in an effort to generate a better understanding of the influencing factors of truth-telling nationwide in Tanzania.

Acknowledgements. The authors are grateful to the Muhimbili University of Health and Allied Sciences, and the management and staff of the ORCI for their tireless support during field work and data collection. Also, sincere thanks go to Rebecca J DeBoer, clinical instructor, Division of Hematology/Oncology, University of California, San Francisco (UCSF), and the patients from the Ocean Road Cancer Institute who volunteered to provide information for this study. Author contributions. RA and GF were involved in the planning of the study, data collection, analysis and interpretation, and preparation of this manuscript. RSJ participated in commentary on the first and subsequent drafts of this manuscript. All authors read and approved the final manuscript.

Funding. None.

Conflicts of interest. None.

1. Limentani AE. The role of ethical principles in health care and the implications for ethical codes. J Med Ethics 1999;25(5):394-398. https://doi. org/10.1136\%2Fjme.25.5.394

2. United Republic of Tanzania. National Cancer Treatment Guidelines. 1st edition. 2020.

3. Beauchamp TL, Walters L, Kahn JP, Matrioanni AC (editors). Contemporary Issues in Bioethics. 7th ed. Wadsworth-Canada: Cengage Learning; 2008.

4. Meulenbergs T. Ethical codes: Between theory and health care practice. Med Law 2003;22:251-258.

5. Littleton V, Meemon N, Breen GDM, et al. An ethical analysis of professional codes in health and medical care. Ethics Med 2010;26(1):25-47.

6. Henderson B, Dorsey JL. Medical Terminology for Dummies. Hoboken, NJ: John Wiley and Sons, 2008
7. Tuckett, A. Truth-telling in clinical practice and the arguments for and against: A review of the literature. Nurs Ethics 2004:11:5005-5013. https://doi. org/10.1191/0969733004ne7280a

8. Begley A, Blackwood B. Truth-telling versus hope: A dilemma in practice. Int J Nurs Pract 2000;6(1):26-31. https://doi.org/10.1046/j.1440-172x.2000.00178.x

9. Vivian R. Truth telling in palliative care nursing: The dilemmas of collusion. Int $J$ Pall Nurs 2006;12(7):341-348. https://doi.org/10.12968/ijpn.2006.12.7.21612

10. Gautam S, Jain N. Indian culture and psychiatry. Ind J Psychiat 2010;52:309-313. https://doi.org/10.4103\%2F0019-5545.69259

11. Wenrich MD, Curtis JR, Shannon SE, Carline JD, Ambrozy DM, Ramsey PG. Communicating with dying patients within the spectrum of medical care from terminal diagnosis to death. Arch Intern Med 2001;161:868-874. https://doi. org/10.1001/archinte.161.6.868

12. Elizabeth O. Withholding truth from patients. Nurs Stand 2009;23(48):35-40

13. Bozcuk $H$, Erdoğan V, Eken $C$, et al. Does awareness of diagnosis make any difference to quality of life? Support Care Cancer 2014;10(1):51-57. https://doi. org/10.1007/s005200100308

14. Mizuno M, Onishi C, Ouishi F. Truth disclosure of cancer diagnoses and its influence on bereaved Japanese families. Cancer Nurs 2002;25(5):396-403. https://doi.org/10.1097/00002820-200210000-00011

15. Beyene Y. Medical disclosure and refugees. Telling bad news to Ethiopian patients. West J Med 1992;157(3):328-332.

16. Harris JJ, Shao J, Sugarman J. Disclosure of cancer diagnosis and prognosis in Northern Tanzania. Soc Sci Med 2003:56(5):905-913. https://doi.org/10.1016/ s0277-9536(02)00090-4

17. Baxter P. Qualitative case study methodology: Study design and implementation for novice researchers. Qual Rep 2008;13(4):544-559. from https://nsuworks. nova.edu/tqr/vol13/iss4/2 (accessed 25 November 2010).

18. Faulkner A. Communication with patients, families and other professionals. BM 1998;316(10):130-132. https://doi.org/10.1136\%2Fbmj.316.7125.130

19. Back AL, Arnold RM, Baile WF, Tulsky JA, Fryer-Edwards K. Approaching difficult communication tasks in oncology. CA Cancer J Clin 2005;55(3):164-177. https:// doi.org/10.3322/canjclin.55.3.164

20. Cohen JS, Erickson JM. Ethical dilemmas and moral distress in oncology nursing practice. Clin J Oncol Nurs 2006;10(6):775-780. https://doi.org/10.1188/06. cjon. $775-780$

21. Norouzinia R, Aghabarari M, Shiri M, Karimi M, Samami E. Communication barriers perceived by nurses and patients. Glob J Health Sci 2015;8(6):65-74. https://doi. org/10.5539\%2Fgjhs.v8n6p65

22. Chan EA, Jones A, Wong K. The relationships between communication, care and time are intertwined: $A$ narrative inquiry exploring the impact of time on registered nurses' work. J Adv Nurs 2013;69(9):2020-2029. https://doi. org/10.1111/jan.12064

23. Anthony DL, Stablein T. Privacy in practice: Professional discourse about information control in health care. J Health Org Management 2016;30(2):207226. https://doi.org/10.1108/jhom-12-2014-0220

24. Ozdogan M, Artac M, Savas B. Factors related to truth-telling practice of physicians. J Pall Med 2006;9(5). https://doi.org/10.1089/jpm.2006.9.1114

25. Abu RR, Tarabeih M. Truth telling to life-threatened and dying patients in Israel: Can legislation improve it? Revista de Studii Interdisciplinare 2018;17(1-2):68-83.

26. Schattner A, Tal M. Truth telling and patient autonomy: The patient's point of view. Am J Med 2002;113(1):66-69. https://doi.org/10.1016/s0002-9343(02)01127-0

27. Kling S. Truth telling in clinical practice: Is it ever OK to lie to patients? Ethics. Curr Allerg Clin Immunol 2012;25(1):34-36.

28. Beste J. Instilling hope and respecting patient autonomy: Reconciling apparently conflicting duties. Bioethics 2005;19(3):215-231. https://doi.org.10.1111/j.14678519.2005.00438.x

29. Kazdaglis GA, Arnaoutoglou C, Karypidis D, Memekidou G, Spanos G, Papadopoulos $\mathrm{O}$. Disclosing the truth to terminal cancer patients: A discussion of ethical and cultural issues. East Mediterr Health J 2010;16(4):442-447.

30. Soni MK, Cella D. Quality of life and symptom measures in oncology: An overview. Am J Manag Care 2002;8(18 Suppl):S560-573.

31. Rosenberg AR, Starks $H$, Unguru $Y$, Feudtner C, Diekema D. Truth telling in the setting of cultural differences and incurable pediatric illness review. JAMA Pediatr 2017;171(11):1113-1119. https://doi.org/10.1001/ jamapediatrics.2017.2568

32. Kelly CDPO, Urch C, Brown EA. The impact of culture and religion on truth telling at the end of life. 2018;June:3838-3842. https://doi.org/10.1093/ndt/gfr630

33. Zahedi F. The challenge of truth telling across cultures: A case study. J Med Ethics Hist Med 2011:4:11.

34. Zolkefli Y. The ethics of truth-telling in health-care settings. Malays J Med Sci 2018;25(3):135-139. https://doi.org/10.21315\%2Fmjms2018.25.3.14

Accepted 13 July 2020. 